Published in final edited form as:

Curr Neurol Neurosci Rep. ; 18(11): 81. doi:10.1007/s11910-018-0887-6.

\title{
The Gut Microbiota and Dysbiosis in Autism Spectrum Disorders
}

\author{
Heather K. Hughes ${ }^{1,2}$, Destanie Rose ${ }^{1,2}$, Paul Ashwood ${ }^{1,2}$ \\ ${ }^{1}$ Department of Medical Microbiology and Immunology, UC Davis, 2805, 50th Street, Sacramento, \\ CA 95817, USA \\ ${ }^{2}$ The M.I.N.D. Institute, University of California at Davis, Davis, CA, USA
}

\begin{abstract}
Purpose of Review-There is a growing body of evidence indicating the gut microbiota influence neurodevelopment and behavior. The purposes of this review are to provide an overview of studies analyzing the microbiota and their metabolites in autism spectrum disorders (ASD) and to discuss the possible mechanisms of action involved in microbial influence on the brain and behavior.
\end{abstract}

Recent Findings-The microbiota-gut-brain (MGB) axis has been extensively studied in animal models, and it is clear that alterations in the composition of microbiota alter neurological and behavioral outcomes. However, findings in human studies are less abundant. Although there are several studies so far showing altered microbiota (dysbiosis) in ASD, the results are heterogeneous and often contradictory. Intervention studies such as fecal microbiota transplant therapies show promise and lend credence to the involvement of the microbiota in ASD.

Summary-A role for the microbiota in ASD is likely; however, further studies elucidating microbial or metabolomic signatures and mechanisms of action are needed. Future research should focus on intervention studies that can identify specific metabolites and immune mediators that improve with treatment to help identify etiologies and pathological mechanisms of ASD.

\section{Keywords}

Autism; Microbiota; Dysbiosis; Dysregulation; Neurodevelopment; Behavior

\section{Introduction}

Autism spectrum disorders (ASD) are a heterogeneous group of neurodevelopmental disorders characterized by deficits in communication, social interaction, and cognition [1]. ASD have increased significantly in prevalence since they were first identified to a current rate of 1:59, with a preponderance toward boys [1]. No single etiology of ASD has been identified, with current theories suggesting both genetic and environmental contributions. Genetic mechanisms account for approximately 10-20\% of ASD cases [2], leaving us with questions about what might be driving etiology of these disorders. Environmental factors

\footnotetext{
$\bowtie$ Paul Ashwood, pashwood@ucdavis.edu.

Conflict of Interest Heather K. Hughes, Destanie Rose, and Paul Ashwood each declare no potential conflicts of interest.

Human and Animal Rights This article does not contain any studies with human or animal subjects performed by any of the authors.
} 
that have been implicated in increased risk for having a child with ASD include air pollution, pesticide exposure, maternal infections, and/or inflammatory conditions, or antibiotics during pregnancy [3-5]. Immune dysfunction and gastrointestinal (GI) inflammation are also common in individuals with ASD and contribute to severity of behaviors seen in the disorder [6-8]. New data on the role of the gut microbiome in neurodevelopmental disorders has prompted theories of the roles commensal bacteria may play in ASD. The studies discussed in this review have identified significant dysbiosis in children with ASD; however, it is unknown whether the GI dysfunction and dysbiosis is sequelae of the larger disorder or whether they directly contribute to causing ASD.

The gut microbiota consists of the collection of microbes present within the human GI tract, and their collective genome is the gut microbiome [9]. It is generally understood that initial colonization begins at birth through the acquisition of maternal microbiota during vaginal delivery; however, recent research suggests that there may be acquisition of maternal microbiota during gestation [10]. The infant microbiota are supported with breastmilk which is high in human oligosaccharides; however, the composition of early-life microbiota can be altered by delivery methods, hygiene, and feeding practices such as formula feeding [10]. The healthy infant gut is initially dominated by Bifidobacterium and Lactobacillus; however, it is unstable for the first several years of life, throughout weaning and food introduction, then stabilizing to a more "adult-like" composition around age 3 [10]. The dominant phyla of bacteria in the healthy adult gut are Firmicutes and Bacteroidetes, with a smaller portion of the microbiota made up of Actinobacteria, Proteobacteria, and Verrucomicrobia [9]. Within the last decade, our understanding of the microbiota and its importance to health has blossomed, and we now recognize that loss or alteration of microbiota may be leading to chronic diseases [11]. Disturbances of the microbiota can occur through antibiotic treatment, changes in diet, immune challenges, and stress [10], and this can upset the balance between beneficial commensals and potentially pathogenic microbes in the gut. This broken balance is termed dysbiosis [12].

\section{Microbiota-Gut-Brain Axis}

The gut microbiota play a critical role in the development of the intestinal architecture and mucosal immune system and are particularly important for regulation of the immune system in the gut [reviewed in [13, 14]]. In germ-free (GF) mice, the architecture of the mesenteric lymph nodes and Peyers patches is smaller and disorganized, with reduced maturation of isolated lymphoid follicles and fewer numbers of immune cells present. These mice experience immune dysfunction and are more susceptible to infections than mice kept in conventional facilities $[13,15]$. These deficits can be corrected by colonization with diverse commensal bacteria; however, this has been shown to be age-dependent, suggesting that both composition and timing of colonization are critical for the education of the immune system [16-18].

The microbiota also play important roles in digestion, nutrient assimilation, vitamin production, and metabolism $[19,20]$ and have recently been shown to have significant influence on the bi-directional signaling that takes place between the gut and the nervous system, termed the microbiota-gut-brain axis [21]. The gut microbiota can influence brain 
function and lead to changes in behaviors, as shown extensively in animal models [22•]. For example, GF rodent studies have shown that microbiota can positively impact stress responses through the hypothalamus-pituitary-adrenal axis (Fig. 1) [23], such as decreased anxiety and increased exploratory behaviors [24-27]. Probiotics have also been shown to increase exploratory behavior in rodents under varied conditions [28-30] including probiotic treatment after dextran sodium sulfate (DSS)-induced colitis [31]. Conversely, stress in early life can induce dysbiosis and influence immune responses, as shown in maternal separation models of stress [32]. Deficits in working memory occur in GF mice [33], and emotional behavior and memory can be modulated through the administration of probiotics [34]. The introduction of probiotics influences gene expression in the central nervous system, specifically altering expression of $\gamma$-aminobutyric acid (GABA) receptors in the hippocampus, mediated through stimulation of the vagus nerve [34]. Microbiota have a profound influence over the synthesis and metabolism of serotonin [26, 35•]. Microbiota produce other neuroactive metabolites including GABA by Lactobacillus and Bifidobacteria [36], acetylcholine by Lactobacillus, dopamine by Bacillus and Serratia, and norepinephrine by Escherichia and Saccharomyces, which could enter circulation and directly affect neural processes throughout the body, including the brain (Fig. 1) [37].

The microbiota can also alter the integrity of the intestinal and the blood-brain barriers, specifically due to their production of short-chain fatty acids [38••, 39], and disrupted barrier function could facilitate the translocation of bacterial metabolites and immune mediators from the gut into circulation (Fig. 1), which could lead to activation of microglia as seen in an induced dysbiosis mouse model [40]. Through such animal studies, evidence is mounting that dysbiosis or altered microbiota composition has an impact on neurodevelopment and behavior.

Dysbiosis and impaired intestinal barrier function were seen in a maternal immune activation (MIA) model of autism, with improvements in ASD relevant behavior after treatment with Bacteriodes fragilis [41]. Recently, this model has been shown to be dependent on microbiota that can induce a $\mathrm{T}$ helper $\left(\mathrm{T}_{\mathrm{H}^{-}}\right) 17$ response-dams that lacked certain microbiota could not mount this response, and their offspring did not display the ASD-like behaviors $[42 \bullet, 43 \bullet \cdot$. Other mouse models of ASD such as the BTBR and Shank3 knock-out mice have shown intrinsic dysbiosis that correlated with the behavioral phenotypes seen in these models $[44,45]$. The latter study saw differences specifically in males, with significant alterations in GABA receptor gene expression in the Shank3 KO mice that could be modulated with the introduction of the probiotic Lactobacillus reuteri [45]. In addition, dysbiosis introduced in conventionally housed adult mice also leads to abnormal behaviors, altered barrier functions, and activation of the microglia, the resident immune cells of the brain [40].

\section{Microbiota in ASD}

Evidence of microbial dysbiosis in ASD has been growing in recent years (Table 1). In addition to immune and GI dysfunction that may be linked to dysbiosis, there is some evidence that altering the microbiota in ASD can improve behaviors [46, 79॰]. The earliest studies investigating the relationship of the microbiota and children with ASD proposed that 
excessive antibiotic use led to an overgrowth of spore-forming Clostridium, which researchers hypothesized might be exposing these children to high levels of microbial metabolites that were neurotoxic. This hypothesis was based on an index case where regressive autism appeared to coincide with several rounds of antibiotic use, and subsequent vancomycin treatment significantly improved aspects of behavior. A small pilot study included 11 children with a regressive form of ASD and who had GI symptoms of diarrhea that were treated with oral vancomycin for 8 weeks, followed by 4 weeks of probiotics. Eight of these children ( 73\%) experienced significantly improved impairments in social behavior and communication. However, these improvements did not endure, with most children reverting to their starting behavioral impairments upon removal of the treatment [46]. Assessment of the microbiota in this study found increased Clostridia (supporting the hypothesis) as well as overgrowth of other spore-forming anerobes, microaerophilic bacteria, and several Clostridia species within the gastric and duodenal secretions not seen in controls [47]. Further classification of the microbiota identified increased Clostridia clusters I/IX and C. bolteae (46-fold increase) [48].

These early findings, coupled with the noteworthy numbers of ASD children with gastrointestinal complaints and immune dysfunction, prompted additional research to determine if the microbiota were consistently altered in ASD. Using fluorescence in situ hybridization (FISH) techniques to identify bacteria present in stool samples, Parracho et al. confirmed increased Clostridia, and its presence highly correlated with GI symptoms in ASD children [49]. However, as studies designed to use more in-depth techniques were published, other bacterial species were identified as having differential abundance in ASD. Increased Desulfovibrio and Bacteroides vulgatus were identified in children with ASD and were correlated to autism severity [50]. Desulfovibrio was also elevated in a small cohort of Slovakian children with ASD and GI dysfunction compared to healthy controls [76].

Desulfovibrio could be an important contributor to GI inflammation, as its major metabolic byproduct—hydrogen sulfide-is cytotoxic to colonic epithelial cells [81]. Moreover, when given to rodents, Desulfovibrio decreased working memory [82].

Ileal and cecal biopsies from children with ASD and GI dysfunction showed increased Firmicutes and decreased Bacteroidetes [51]. As part of this study, the researchers looked at gene expression associated with carbohydrate digestion and transport and found impaired expression of these genes correlated with dysbiosis in ASD [51]. Dysfunctional carbohydrate digestion could alter the fermentation byproducts of the microbiota present, and undigested carbohydrate could preferentially select for certain bacteria [51]. Kang et al. found that children with ASD and GI dysfunction had decreased in commensals important for carbohydrate fermentation including Prevotella, Coprococcus, and unclassified Veillonellaceae [74]. A more recent biopsy study examined duodenal samples of ASD children with GI dysfunction to determine if disaccharidase activity was altered compared to controls, similar to the previous study. Although they did not see overall differences in diversity, they identified elevations in Burkholderia and reduced Prevotella and Neisseria in the duodenum of ASD children. Overall, they did not see the same reductions in disaccharidases; however, they found a correlation between disaccharidase activity with the presence of Clostridium [77]. 
Increased Sutterella, a mucosa-associated microbe, was found in significant numbers in intestinal biopsies of ASD children with GI dysfunction [56]. Sutterella was also increased in stool samples of ASD children, irrespective of GI issues [80]. Increased Ruminococcus torques [80] was also seen in ASD and is similar to dysbiosis noted in inflammatory bowel disorders (IBD) [71]. As well as increases in some species, reductions in important commensals Bifidobacteria and the mucin-degrading Akkermansia muciniphila [52] have been shown in ASD. One of the largest of recent studies that looked at 40 ASD (36 severe) and 40 typically developing control children found decreased abundance of the Alistipes, Bilophila, Dialister, Parabacteroides, and Veillonella families but increased Collinsella, Corynebacterium, Dorea, and Lactobacillus, suggesting major changes in the microbiota composition in ASD. The authors also found that constipation in ASD correlated with increases in Escherichia/Shigella and Clostridium cluster XVIII, a cluster known to produce exotoxins that are pro-inflammatory [78].

Two recent studies have provided evidence to support the hypothesis that children with ASD and GI dysfunction have elevated inflammatory immune responses and associated dysbiosis $[57,83]$. Notably, the balance of inflammatory cytokines was skewed in children with ASD and GI dysfunction when compared with regulatory cytokines such as transforming growth factor (TGF) beta 1 [57]. However, whether it is the push towards an inflammatory environment that influences the microbiota composition in ASD or a bacterial composition that elicits inflammation needs further study. Inflammatory conditions in the GI tract are known to exacerbate dysbiosis, for example, during antibiotic-associated inflammation, increases in host-derived nitrate encourage Proteobacteria such as Escherichia coli to bloom $[84,85]$. Differences in microbiota diversity, both at the family level and when comparing functional KEGG pathways, were also seen in children with ASD compared to typically developing controls. Interestingly, altered zonulin levels were seen in ASD children with GI dysfunction and may suggest increased intestinal permeability [57]. Luna et al. also showed that increased cytokines associated with mucosal immunity, including interleukin [IL]6, IL-1, IL-17A, and interferon (IFN)-gamma, were associated with abdominal pain and increased Clostridiales in ASD [83]. A recent clinical trial which involved fecal microbiota transplant (FMT) in 18 ASD children led to significantly improved GI symptoms and ASDrelevant behaviors, with increases in Bifidobacterium, Prevotella, and Desulfovibrio. These changes persisted at the 8-week follow-up [79•]. Since immune dysfunction and cytokine dysregulation are so prominent in ASD [reviewed in $[6,8,86]$, measuring immune responses and inflammatory cytokines in future probiotic and FMT clinical trials could help identify the role that dysbiosis plays in this dysfunction.

Overall, there does not seem to be consensus for differences in microbiota in ASD, and a meta-analysis of 15 studies could not amalgamate the often contradictory results [87]. Some studies found increased diversity of the microbiota in ASD [47, 64], whereas other studies show the opposite trend towards decreased diversity and richness [73,74], or no differences in diversity [75, 77]. Several studies found an increased Firmicutes/Bacteroidetes ratio in both stool and biopsy samples, including one study that showed probiotic treatment reversed this trend [51, 76, 78]. However, other studies noted an opposite trend in stool [50, 64]. Tissue/specimen type and sample site may account for the differences in studies. For example, when fecal samples are analyzed, this will only detect what has moved through the 
GI tract, not necessarily the dominant colonizers of the varied regions throughout the GI tract. This may skew results to those species that have difficulty adhering to the intestinal epithelium or species that are not surviving well within the GI tract and may explain some of the opposing trends seen when comparing studies using stools versus biopsy specimens [51]. Other technical issues may relate to comparing profiles to siblings who may also exhibit broader behavioral impairments rather than typically developing controls. Siblings are also more likely to have shared a common environment that would alter the microbiota $[55,75]$. Additional sources of error include differing techniques; for example, sequencing can vary in error profile, and results can vary based on read length and sequencing depth [88]. Heterogeneity in age of subjects and sex may also reduce comparability across studies. Future studies should investigate microbiota profiles at earlier time points pre-diagnoses of ASD to help determine if there are differences between microbiota and how that relates to ASD outcome or broader neurodevelopmental concerns such as anxiety and cognition. Note that the composition of microbiota is very sensitive to changes diet, fiber composition, etc., and can also be altered by stress and other compounding factors [10]. Children with ASD are known to have feeding problems, food sensitivities/aversions, and extremely restricted diets [89], which could also be contributing to differences in microbiota but not to an ASD diagnosis. A preliminary study showed that despite no differences in the composition of microbiota, early-life probiotic treatment and presence of Bifidobacterium during infancy reduced the risk of neurodevelopmental disorders [90]. This early period of colonization is an area needing further research with larger studies and advanced technological tools.

Bacteria are not the only microbes to make up the microbiota. New techniques are identifying the fungal microbiota and its role in human health [91]. GI overgrowth of fungal species such as Candida may have deleterious immune consequences and have been linked to IBD and celiac disease [92-94]. Fungal commensals can bloom after antibiotic administration, and the presence of Candida can interfere with reassembly of the microbiota after antibiotic perturbation, contributing to dysbiosis [95]. The immune system responds to fungal infections with a $\mathrm{T}_{\mathrm{H}^{-}} 17$ response, producing IL-17, a cytokine recently implicated in ASD etiology in the MIA mouse model [42•, 43••]. So far, fungal microbiota studies in ASD are scant; however, one culture-based study showed significant presence of Candida species in the feces of children with ASD, the majority of which were Candida albicans.

Fluconozole-resistant species were also found in significant numbers of cultures, including C. krusei and C. glabrata [96]. A separate study found Candida present in nearly $60 \%$ of ASD samples, with none present in controls. They also identified hyphae formation, suggesting that the dimorphic yeast had switched to its invasive and adhesive form [97]. However, one culture-based study looked for the presence of yeast in ASD children and did not see an over-representation compared to control samples [53]. Newer studies that utilize sequencing techniques may be more reliable and have shown that Candida is the most abundant taxa of mycobiota seen in children with ASD, found at nearly twice the rate of typically developed children [78]. Elevated ratios of the urinary metabolites D-arabinitol/Larabinitol (DA/LA) have been used for early identification of invasive candidiasis, as darabinitol is a major metabolite of pathogenic fungal species [98]. A preliminary study of 22 children with ASD and GI dysfunction found that DA concentrations and DA/LA ratios were significantly reduced with daily administration of probiotics, and improvements were 
seen in certain ASD behaviors including ability to concentrate [62]. Elevated DA (listed as D-arabitol) was also found in a 2014 study of 21 Italian children with ASD [67]. This group also saw elevated glycolic acid, which may also be associated with overgrowth of yeast in the GI tract [67].

\section{Microbial Metabolites in ASD}

Due to the many limitations of microbiota analysis, some researchers have turned to metabolomic tools to identify how byproducts of microbial fermentation and metabolism might be interacting with human health or influencing disease. These tools identify altered patterns of metabolites within urine, stool, and blood samples that may provide a biochemical signature of ASD and supportive evidence of dysbiotic gut microbiota [99]. A summary of metabolomics studies specific to the microbiota and ASD are listed in Table 2.

Altered patterns of bacterial metabolites were seen in a large number of children with ASD compared to healthy control children. These metabolites included elevated 3-(3hydroxyphenyl)-3-hydroxypropionic acid (HPHPA), a catabolic byproduct of Clostridia [59]. Increased HPHPA was found more recently in study of Italian children with ASD [67], and a 2016 intervention study showed that Vancomycin treatment could reverse increases of HPHPA and two associated metabolites [69]. Reduced hippurate, phenyacetylglutamine, and p-cresylsulfate were seen in ASD children, indicating alterations in gut microbiota [58]. Pcresol, another microbial metabolite produced by Clostridium species, was significantly elevated in children with ASD, especially females with severe autism [60]. Further studies showed that elevations of urinary p-cresol and other derivative metabolites of Clostridia were associated with repetitive behaviors [65] and constipation in ASD [70]. In animal models of ASD, elevations of microbial metabolite 4-ethylphenylsulfate (4EPS) correlated with altered behaviors in mice, and probiotic therapy reduced its concentration and improved behaviors in MIA model of ASD [41]. 4EPS is chemically related to p-cresol, the uremic toxin seen elevated in ASD children [41, 60, 65].

Short-chain fatty acids (SCFA) are microbial metabolites of fiber fermentation and can be found in high concentrations in the colon. SCFA metabolites of commensal microbiota are generally beneficial to the host; however, some SCFAs such as propionic acid (PPA) can be neurotoxic in higher concentrations and have been shown to cause behavioral abnormalities in rodent models [100, 101] (Fig. 1). SCFA influence gene expression epigenetically through histone deacetylase (HDAC) inhibition. For example, butyrate-producing bacteria promote peripheral regulatory $\mathrm{T}$ cell $\left(\mathrm{T}_{\text {regs }}\right)$ expansion, altering gut immunity by promoting tolerance. This occurs through HDAC inhibition leading to increased acetylation at the Foxp3 promotor and expansion of $\mathrm{CD}^{+}{ }^{+}$Foxp $^{+} \mathrm{T}_{\text {regs }}[102 \bullet \bullet]$. Therefore, shifts in SCFA production could potentially contribute to altered immune regulation in the gut and lead to peripheral inflammation (Fig. 1). Several studies found lower levels of fecal SCFA, including butyrate in feces of ASD children [53, 64], while a 2012 study found increased ammonia and SCFA including acetic, butyric, isobutyric, valeric, and isovaleric acids in ASD [61]. Lower SCFA occurred alongside elevated phenol, 4-(1,1-dimethylethyl)-phenol and p-cresol, and correlated with increased Bacteroides and Clostridia and decreased 
Ruminococcoceae [64]. These children also had elevations in free amino acids, indicating increases in proteolytic bacteria [64].

Tryptophan (Trp) metabolites directly influence host physiology including immune and gut homeostasis [reviewed in: [103]]. The gut microbiota are critical for regulating Trp metabolism, either directly by degrading Trp to indole-derivatives or indirectly through mammalian kynurenine and serotonin pathways. GF mice have increased levels of circulating Trp, adding evidence to the importance of the microbiota for Trp metabolism [26, 104]. Metabolome studies have repeatedly shown evidence of alterations in tryptophan, kynurenine, and serotonin pathways in children with ASD, including reports of increased urinary excretion of tryptophan and associated metabolites [63, 67, 68, 72, 105]. The primary metabolic pathway of tryptophan is the kynurenine pathway, leading to production of kynurenic acid. Increases in an alternative branch of the kynurenine pathway led to reduced kynurenic acid and elevated quinolinic acid (QA), a compound known to be an excitotoxic N-methyl-D-aspartate (NMDA) receptor agonist, in children with ASD [68] (Fig. 1). Immune activation may be responsible for this elevation, as activated macrophages and microglia are the main producers of QA [106]. Bacterial degradation of tryptophan, yielding increased indolyl 3-acetic acid, indolyl lactate, and other indole derivatives were also shown in ASD and these shifts were associated with reduced urinary melatonin, downstream of serotonin production that may be the result of bacterial metabolism of available tryptophan [68]. In 2014, a meta-analysis of 22 studies that measured alterations in blood serotonin concluded that there was significantly elevated blood and platelet-rich plasma 5-HT in ASD individuals compared to controls [66]. Serotonergic metabolites were also increased with a decrease in Trp in mucosal biopsies of children with ASD and functional GI disorders, and these findings strongly correlated with several specific bacteria (Table 2) [83]. Spore-forming gut microbiota mediate the production of peripheral serotonin (5-HT) from Trp through the production of metabolites that increase expression of tryptophan hydroxylase (Tph) 1 in enterochromaffin (EC) cells of the gut which in turn increases colonic and circulating levels of 5-HT [35•]. Serotonin expressed in enteric neurons early in development can also contribute to the 5-HT pool and motility of the GI tract [107]. GF mouse models have shown that the serotonergic system in the brain is also influenced by the microbiota [24, 26], whereby excess peripheral Trp may cross the BBB to influence the rate serotonin synthesis in the brain [108]. Serotonin plays important neurotrophic roles during early development, and elevations of 5-HT during critical time periods can alter cognition and sensory processing [109].

Microbial metabolites and inflammatory mediators are capable of signaling through the vagus nerve [110] (Fig. 1). As the major nerve of the parasympathetic nervous system, it has a bidirectional role in enervating organs throughout the body including the gut. The majority of the fibers of the vagus nerve are afferent sensory fibers, delivering sensory information from the periphery, including the GI tract, to the brain [110]. It is understood that neural communication between the brain and the microbiota may be occurring indirectly through hormones or neurotransmitter release by gut endocrine cells in presence of the microbiota and metabolites, or perhaps through direct pattern recognition sensing due to Toll-like receptor expression on afferent fibers [110]. Vagal activation by the microbiota has been shown in several animal models: vagotomy caused reversal of probiotic effects on memory 
and hippocampal GABA receptor gene expression [34], and also attenuated anxiety in mice with DSS-induced colitis. Administration of Bifidobacterium longum also reduced anxiety; however, vagotomy prevented this probiotic effect [111]. Although vagal involvement has been identified in some studies, others show independence of vagal communication. For example, alteration of the microbiota increased exploratory behavior in mice and increased brain-derived neurotrophic factor in the hippocampus. These effects were not influenced by vagotomy [112]. More research is needed to elucidate this mechanism further.

\section{Conclusion}

Why do children with ASD have dysbiosis? Is this inherent to the disorder, or perhaps causal? These significant questions still remain to be determined. Immune system dysfunction is a well-known issue in ASD, possibly driving the dysbiotic microbiota, or alternatively created by it. The relationship between dysbiosis and the high incidence of comorbid GI dysfunction in ASD is not well elucidated; however, these studies reviewed here indicate that a relationship exists. Reports that behaviors improve after modification of the microbiota support the hypothesis that dysbiotic microbiota, their influence on the immune system, and their metabolic byproducts contribute directly to the development of these disorders. More research to clarify mechanism(s) of the influence of dysbiosis on brain and immune function and behavior are needed.

\section{Acknowledgements}

Investigators were funded by the NIEHS Children's Center grant (P01 ES011269), US EPA STAR program grant (R833292 and R829388), NIEHS CHARGE study (R01ES015359), NICHD (HD086669, HD090214 and U54 HD079125), Autism Research Institute, Autism Speaks Foundation, The Boler Company Foundation, NARSAD Foundation and the Johnson Foundation. This material is based upon work supported by the National Science Foundation Graduate Research Fellowship under Grant No. 1650042.

\section{References}

Papers of particular interest, published recently, have been highlighted as:

- Of importance

•- Of major importance

1. Baio J, Wiggins L, Christensen DL, Maenner MJ, Daniels J, Warren Z, et al. Prevalence of autism Spectrum disorder among children aged 8 years - autism and developmental disabilities monitoring network, 11 sites, United States, 2014. MMWR Surveill Summ. 2018;67(6):1-23.

2. Abrahams BS, Geschwind DH. Advances in autism genetics: on the threshold of a new neurobiology. Nat Rev Genet. 2008;9(5): 341-55. [PubMed: 18414403]

3. Raz R, Roberts AL, Lyall K, Hart JE, Just AC, Laden F, et al. Autism spectrum disorder and particulate matter air pollution before, during, and after pregnancy: a nested case-control analysis within the Nurses' health study II cohort. Environ Health Perspect. 2015;123(3):264-70. [PubMed: 25522338]

4. Shelton JF, Geraghty EM, Tancredi DJ, Delwiche LD, Schmidt RJ, Ritz B, et al. Neurodevelopmental disorders and prenatal residential proximity to agricultural pesticides: the CHARGE study. Environ Health Perspect. 2014;122(10):1103-9. [PubMed: 24954055]

5. Atladóttir HÓ, Henriksen TB, Schendel DE, Parner ET. Autism after infection, febrile episodes, and antibiotic use during pregnancy: an exploratory study. Pediatrics. 2012;130. 
6. Onore C, Careaga M, Ashwood P. The role of immune dysfunction in the pathophysiology of autism. Brain Behav Immun. 2012;26(3):383-92. [PubMed: 21906670]

7. McElhanon BO, McCracken C, Karpen S, Sharp WG. Gastrointestinal symptoms in autism spectrum disorder: a meta-analysis. Pediatrics. 2014;133(5):872-83. [PubMed: 24777214]

8. Mead J, Ashwood P. Evidence supporting an altered immune response in ASD. Immunol Lett. 2015;163(1):49-55. [PubMed: 25448709]

9. The Human Microbiome Project C. Structure, function and diversity of the healthy human microbiome. Nature. 2012;486:207. [PubMed: 22699609]

10. RodrÍguez JM, Murphy K, Stanton C, Ross RP, Kober OI, Juge N, et al. The composition of the gut microbiota throughout life, with an emphasis on early life. Microb Ecol Health Dis. 2015;26(1): 26050. [PubMed: 25651996]

11. Blaser MJ, Falkow S. What are the consequences of the disappearing human microbiota? Nat Rev Microbiol. 2009;7(12): 887-94. [PubMed: 19898491]

12. Tamboli CP, Neut C, Desreumaux P, Colombel JF. Dysbiosis in inflammatory bowel disease. Gut. 2004;53(1):1-4. [PubMed: 14684564]

13. Belkaid Y, Hand TW. Role of the microbiota in immunity and inflammation. Cell. 2014;157(1): 121-41. [PubMed: 24679531]

14. Hooper LV, Littman DR, Macpherson AJ. Interactions between the microbiota and the immune system. Science. 2012;336(6086): 1268-73. [PubMed: 22674334]

15. Round JL, Mazmanian SK. The gut microbiome shapes intestinal immune responses during health and disease. Nature reviews. Immunology 2009;9(5):313-323.

16. Chung H, Pamp SJ, Hill JA, Surana NK, Edelman SM, Troy EB, et al. Gut immune maturation depends on colonization with a host-specific microbiota. Cell. 2012;149(7):1578-93. [PubMed: 22726443]

17. El Aidy S, Hooiveld G, Tremaroli V, Backhed F, Kleerebezem M. The gut microbiota and mucosal homeostasis: colonized at birth or at adulthood, does it matter? Gut Microbes 2013;4(2): 118-124. [PubMed: 23333858]

18. Gensollen T, Iyer SS, Kasper DL, Blumberg RS. How colonization by microbiota in early life shapes the immune system. Science. 2016;352(6285):539-44. [PubMed: 27126036]

19. van de Wouw M, Schellekens H, Dinan TG, Cryan JF. Microbiota-gut-brain Axis: modulator of host metabolism and appetite. J Nutr. 2017;147(5):727-45. [PubMed: 28356427]

20. Jandhyala SM, Talukdar R, Subramanyam C, Vuyyuru H, Sasikala M, Reddy DN. Role of the normal gut microbiota. World J Gastroenterol: WJG. 2015;21(29):8787-803. [PubMed: 26269668]

21. Cryan JF, Dinan TG. Mind-altering microorganisms: the impact of the gut microbiota on brain and behaviour. Nat Rev Neurosci. 2012;13(10):701-12. [PubMed: 22968153]

22•. Vuong HE, Yano JM, Fung TC, Hsiao EY. The microbiome and host behavior. Ann Rev Neurosci. 2017;40(1):21-49 [PubMed: 28301775] Comprehensive review of the influence of microbiota on behavior in animal models.

23. Sudo N, Chida Y, Aiba Y, Sonoda J, Oyama N, Yu XN, et al. Postnatal microbial colonization programs the hypothalamic-pituitary-adrenal system for stress response in mice. J Physiol. 2004;558(Pt 1):263-75. [PubMed: 15133062]

24. Neufeld KM, Kang N, Bienenstock J, Foster JA. Reduced anxiety-like behavior and central neurochemical change in germ-free mice. Neurogastroenterol Motil. 2011;23(3):255-64 e119. [PubMed: 21054680]

25. Diaz Heijtz R, Wang S, Anuar F, Qian Y, Bjorkholm B, Samuelsson A, et al. Normal gut microbiota modulates brain development and behavior. Proc Natl Acad Sci U S A. 2011;108(7): 3047-52. [PubMed: 21282636]

26. Clarke G, Grenham S, Scully P, Fitzgerald P, Moloney RD, Shanahan F, et al. The microbiome-gutbrain axis during early life regulates the hippocampal serotonergic system in a sex-dependent manner. Mol Psychiatry. 2013;18(6):666-73. [PubMed: 22688187]

27. Campos AC, Rocha NP, Nicoli JR, Vieira LQ, Teixeira MM, Teixeira AL. Absence of gut microbiota influences lipopolysaccharide-induced behavioral changes in mice. Behav Brain Res. 2016;312:186-94. [PubMed: 27316342] 
28. Matthews DM, Jenks SM. Ingestion of Mycobacterium vaccae decreases anxiety-related behavior and improves learning in mice. Behav Process. 2013;96:27-35.

29. Savignac HM, Tramullas M, Kiely B, Dinan TG, Cryan JF. Bifidobacteria modulate cognitive processes in an anxious mouse strain. Behav Brain Res. 2015;287:59-72. [PubMed: 25794930]

30. Liang S, Wang T, Hu X, Luo J, Li W, Wu X, et al. Administration of Lactobacillus helveticus NS8 improves behavioral, cognitive, and biochemical aberrations caused by chronic restraint stress. Neuroscience. 2015;310:561-77. [PubMed: 26408987]

31. Emge JR, Huynh K, Miller EN, Kaur M, Reardon C, Barrett KE, et al. Modulation of the microbiota-gut-brain axis by probiotics in a murine model of inflammatory bowel disease. Am $\mathbf{J}$ Physiol Gastrointest Liver Physiol. 2016;310(11):G989-98. [PubMed: 27056723]

32. O'Mahony SM, Marchesi JR, Scully P, Codling C, Ceolho AM, Quigley EM, et al. Early life stress alters behavior, immunity, and microbiota in rats: implications for irritable bowel syndrome and psychiatric illnesses. Biol Psychiatry. 2009;65(3):263-7.

33. Gareau MG, Wine E, Rodrigues DM, Cho JH, Whary MT, Philpott DJ, et al. Bacterial infection causes stress-induced memory dysfunction in mice. Gut. 2011;60(3):307-17. [PubMed: 20966022]

34. Bravo JA, Forsythe P, Chew MV, Escaravage E, Savignac HM, Dinan TG, et al. Ingestion of lactobacillus strain regulates emotional behavior and central GABA receptor expression in a mouse via the vagus nerve. Proc Natl Acad Sci USA. 2011;108(38): 16050-5. [PubMed: 21876150]

35•. Yano JM, Yu K, Donaldson GP, Shastri GG, Ann P, Ma L, et al. Indigenous bacteria from the gut microbiota regulate host serotonin biosynthesis. Cell. 2015;161(2):264-76 [PubMed: 25860609] Elegant study showing that serotonin production by the enterochromaffin cells in the gut is significantly influenced by spore-forming gut microbiota.

36. Barrett E, Ross RP, O’Toole PW, Fitzgerald GF, Stanton C. Gamma-aminobutyric acid production by culturable bacteria from the human intestine. J Appl Microbiol. 2012;113(2):411-7. [PubMed: 22612585]

37. Lyte M Probiotics function mechanistically as delivery vehicles for neuroactive compounds: microbial endocrinology in the design and use of probiotics. BioEssays. $2011 ; 33(8): 574-81$. [PubMed: 21732396]

38••. Braniste V, Al-Asmakh M, Kowal C, Anuar F, Abbaspour A, Toth M, et al. The gut microbiota influences blood-brain barrier permeability in mice. Sci Transl Med. 2014;6(263):263ra158Study shows the influence of gut microbiota on the integrity of the blood-brain barrier, and found that mono-colonization with short-chain fatty acid producing bacteria could decrease blood-brain barrier permeability.

39. Kelly CJ, Zheng L, Campbell EL, Saeedi B, Scholz CC, Bayless AJ, et al. Crosstalk between microbiota-derived short-chain fatty acids and intestinal epithelial HIF augments tissue barrier function. Cell Host Microbe. 2015;17(5):662-71. [PubMed: 25865369]

40. Bruce-Keller AJ, Salbaum JM, Luo M. Blanchard et, Taylor CM, welsh DA, et al. obese-type gut microbiota induce neurobehavioral changes in the absence of obesity. Biol Psychiatry. 2015;77(7): 607-15. [PubMed: 25173628]

41. Hsiao EY, McBride SW, Hsien S, Sharon G, Hyde ER, McCue T, et al. Microbiota modulate behavioral and physiological abnormalities associated with neurodevelopmental disorders. Cell. 2013;155(7):1451-63. [PubMed: 24315484]

42•. Kim S, Kim H, Yim YS, Ha S, Atarashi K, Tan TG, et al. Maternal gut bacteria promote neurodevelopmental abnormalities in mouse offspring. Nature. 2017;549(7673):528-32 [PubMed: 28902840] Choi et al., 2016 identified the role of IL-17 in a maternal activation model of ASD. This follow up study study found that maternal IL-17 production was dependent on the composition of maternal microbiota.

43••. Choi GB, Yim YS, Wong H, Kim S, Kim H, Kim SV, et al. The maternal interleukin-17a pathway in mice promotes autism-like phenotypes in offspring. Science. 2016;351(6276):933-9 [PubMed: 26822608] Important study identifying the role of maternal IL-17 in abnormal cortical development in the offspring of a maternal activation model of autism. 
44. Golubeva AV, Joyce SA, Moloney G, Burokas A, Sherwin E, Arboleya S, et al. Microbiota-related changes in Bile Acid \& Tryptophan Metabolism are associated with gastrointestinal dysfunction in a mouse model of autism. EBioMedicine. 2017;24: 166-78. [PubMed: 28965876]

45. Tabouy L, Getselter D, Ziv O, Karpuj M, Tabouy T, Lukic I, et al. Dysbiosis of microbiome and probiotic treatment in a genetic model of autism spectrum disorders. In: Brain Behav Immun; 2018.

46. Sandler RH, Finegold SM, Bolte ER, Buchanan CP, Maxwell AP, Vaisanen ML, et al. Short-term benefit from oral vancomycin treatment of regressive-onset autism. J Child Neurol. 2000;15(7): 429-35. [PubMed: 10921511]

47. Finegold SM, Molitoris D, Song Y, Liu C, Vaisanen ML, Bolte E, et al. Gastrointestinal microflora studies in late-onset autism. Clin Infect Dis. 2002;35(Suppl 1):S6-s16. [PubMed: 12173102]

48. Song Y, Liu C, Finegold SM. Real-time PCR quantitation of clostridia in feces of autistic children. Appl Environ Microbiol. 2004;70(11):6459-65. [PubMed: 15528506]

49. Parracho HM, Bingham MO, Gibson GR, McCartney AL. Differences between the gut microflora of children with autistic spectrum disorders and that of healthy children. J Med Microbiol. 2005;54(Pt 10):987-91. [PubMed: 16157555]

50. Finegold SM, Dowd SE, Gontcharova V, Liu C, Henley KE, Wolcott RD, et al. Pyrosequencing study of fecal microflora of autistic and control children. Anaerobe. 2010;16(4):444-53. [PubMed: 20603222]

51. Williams BL, Hornig M, Buie T, Bauman ML, Cho Park M, Wick I, et al. Impaired carbohydrate digestion and transport and mucosal dysbiosis in the intestines of children with autism and gastrointestinal disturbances. PLoS One. 2011;6(9):e24585. [PubMed: 21949732]

52. Wang L, Christophersen CT, Sorich MJ, Gerber JP, Angley MT, Conlon MA. Low relative abundances of the mucolytic bacterium Akkermansia muciniphila and Bifidobacterium spp. in feces of children with autism. Appl Environ Microbiol. 2011;77(18): 6718-21. [PubMed: 21784919]

53. Adams JB, Johansen LJ, Powell LD, Quig D, Rubin RA. Gastrointestinal flora and gastrointestinal status in children with autism-comparisons to typical children and correlation with autism severity. BMC Gastroenterol. 2011;11:22. [PubMed: 21410934]

54. Martirosian G, Ekiel A, Aptekorz M, Wiechula B, Kazek B, Jankowska-Steifer E, et al. Fecal lactoferrin and Clostridium spp. in stools of autistic children. Anaerobe. 2011;17(1):43-5. [PubMed: 21167951]

55. Gondalia SV, Palombo EA, Knowles SR, Cox SB, Meyer D, Austin DW. Molecular characterisation of gastrointestinal microbiota of children with autism (with and without gastrointestinal dysfunction) and their neurotypical siblings. Autism Res. 2012;5(6):419-27. [PubMed: 22997101]

56. Williams BL, Hornig M, Parekh T, Lipkin WI. Application of novel PCR-based methods for detection, quantitation, and phylogenetic characterization of Sutterella species in intestinal biopsy samples from children with autism and gastrointestinal disturbances. MBio. 2012;3(1).

57. Rose DR, Yang H, Serena G, Sturgeon C, Ma B, Careaga M, et al. Differential immune responses and microbiota profiles in children with autism spectrum disorders and co-morbid gastrointestinal symptoms. Brain. Behavior, and Immunity. 2018;70:354-68.

58. Yap IK, Angley M, Veselkov KA, Holmes E, Lindon JC, Nicholson JK. Urinary metabolic phenotyping differentiates children with autism from their unaffected siblings and age-matched controls. J Proteome Res. 2010;9.

59. Shaw W Increased urinary excretion of a 3-(3-hydroxyphenyl)-3-hydroxypropionic acid (HPHPA), an abnormal phenylalanine metabolite of clostridia spp. in the gastrointestinal tract, in urine samples from patients with autism and schizophrenia. Nutr Neurosci. 2010;13(3):135-43. [PubMed: 20423563]

60. Altieri L, Neri C, Sacco R, Curatolo P, Benvenuto A, Muratori F, et al. Urinary p-cresol is elevated in small children with severe autism spectrum disorder. Biomarkers: biochemical indicators of exposure, response, and susceptibility to chemicals. Biomarkers 2011 ;16(3): 252-60. [PubMed: 21329489] 
61. Wang L, Christophersen CT, Sorich MJ, Gerber JP, Angley MT, Conlon MA. Elevated fecal short chain fatty acid and ammonia concentrations in children with autism spectrum disorder. Dig Dis Sci. 2012;57(8):2096-102. [PubMed: 22535281]

62. Kaluzna-Czaplinska J, Blaszczyk S. The level of arabinitol in autistic children after probiotic therapy. Nutrition (Burbank, Los Angeles County, Calif) 2012;28(2):124-6.

63. Ming X, Stein TP, Barnes V, Rhodes N, Guo L. Metabolic perturbance in autism spectrum disorders: a metabolomics study. J Proteome Res. 2012;11(12):5856-62. [PubMed: 23106572]

64. De Angelis M, Piccolo M, Vannini L, Siragusa S, De Giacomo A, Serrazzanetti DI. Fecal microbiota and metabolome of children with autism and pervasive developmental disorder not otherwise specified. PLoS One 2013;8.

65. Gabriele S, Sacco R, Cerullo S, Neri C, Urbani A, Tripi G, et al. Urinary p-cresol is elevated in young French children with autism spectrum disorder: a replication study. Biomarkers : biochemical indicators of exposure, response, and susceptibility to chemicals. Biomarkers 2014;19(6):463-70. [PubMed: 25010144]

66. Gabriele S, Sacco R, Persico AM. Blood serotonin levels in autism spectrum disorder: a systematic review and meta-analysis. Eur Neuropsychopharmacol. 2014;24(6):919-29. [PubMed: 24613076]

67. Noto A, Fanos V, Barberini L, Grapov D, Fattuoni C, Zaffanello M, et al. The urinary metabolomics profile of an Italian autistic children population and their unaffected siblings. $\mathrm{J}$ Matern Fetal Neonatal Med. 2014;27(sup2):46-52. [PubMed: 25284177]

68. Gevi F, Zolla L, Gabriele S, Persico AM. Urinary metabolomics of young Italian autistic children supports abnormal tryptophan and purine metabolism. Molecular Autism. 2016;7:47. [PubMed: 27904735]

69. Xiong X, Liu D, Wang Y, Zeng T, Peng Y. Urinary 3-(3-Hydroxyphenyl)-3-hydroxypropionic acid, 3-Hydroxyphenylacetic acid, and 3-Hydroxyhippuric acid are elevated in children with autism Spectrum disorders. Biomed Res Int. 2016;2016:9485412. [PubMed: 27123458]

70. Gabriele S, Sacco R, Altieri L, Neri C, Urbani A, Bravaccio C, et al. Slow intestinal transit contributes to elevate urinary p-cresol level in Italian autistic children. Autism Res. 2016;9(7):7529. [PubMed: 26437875]

71. Png CW, Linden SK, Gilshenan KS, Zoetendal EG, McSweeney CS, Sly LI, et al. Mucolytic bacteria with increased prevalence in IBD mucosa augment in vitro utilization of mucin by other bacteria. Am J Gastroenterol. 2010;105(11):2420-8. [PubMed: 20648002]

72. Lussu M, Noto A, Masili A, Rinaldi AC, Dessi A, De Angelis M, et al. The urinary 1H-NMR metabolomics profile of an italian autistic children population and their unaffected siblings. Autism Res. 2017;10(6):1058-66. [PubMed: 28296209]

73. Kang D-W, Ilhan ZE, Isern NG, Hoyt DW, Howsmon DP, Shaffer M, et al. Differences in fecal microbial metabolites and microbiota of children with autism spectrum disorders. Anaerobe. 2018;49: 121-31. [PubMed: 29274915]

74. Kang DW, Park JG, Ilhan ZE, Wallstrom G, Labaer J, Adams JB, et al. Reduced incidence of Prevotella and other fermenters in intestinal microflora of autistic children. PLoS One. 2013;8(7): e68322. [PubMed: 23844187]

75. Son JS, Zheng LJ, Rowehl LM, Tian X, Zhang Y, Zhu W, et al. Comparison of fecal microbiota in children with autism Spectrum disorders and Neurotypical siblings in the Simons simplex collection. PLoS One. 2015;10(10):e0137725. [PubMed: 26427004]

76. Tomova A, Husarova V, Lakatosova S, Bakos J, Vlkova B, Babinska K, et al. Gastrointestinal microbiota in children with autism in Slovakia. Physiol Behav. 2015;138:179-87. [PubMed: 25446201]

77. Kushak RI, Winter HS, Buie TM, Cox SB, Phillips CD, Ward NL. Analysis of the duodenal microbiome in autistic individuals: association with carbohydrate digestion. J Pediatr Gastroenterol Nutr. 2017;64(5):e110-e6. [PubMed: 27811623]

78. Strati F, Cavalieri D, Albanese D, De Felice C, Donati C, Hayek J, et al. New evidences on the altered gut microbiota in autism spectrum disorders. Microbiome. 2017;5(1):24. [PubMed: 28222761]

79•. Kang D-W, Adams JB, Gregory AC, Borody T, Chittick L, Fasano A, et al. Microbiota Transfer Therapy alters gut ecosystem and improves gastrointestinal and autism symptoms: an open-label 
study. Microbiome. 2017;5(1):10 [PubMed: 28122648] Pilot study of 18 children with ASD showed significant and persistent improvements in GI symptoms and ASD-relevant behaviors, with increases in Bifidobacterium, Prevotella and Desulfovibrio.

80. Wang L, Christophersen CT, Sorich MJ, Gerber JP, Angley MT, Conlon MA. Increased abundance of Sutterella spp, and Ruminococcus torques in feces of children with autism spectrum disorder. Mol Autism. 2013;4. [PubMed: 23422139]

81. Carbonero F, Benefiel AC, Alizadeh-Ghamsari AH, Gaskins HR. Microbial pathways in colonic sulfur metabolism and links with health and disease. Front Physiol. 2012;3:448. [PubMed: 23226130]

82. Ritz NL, Burnett BJ, Setty P, Reinhart KM, Wilson MR, Alcock J, et al. Sulfate-reducing bacteria impairs working memory in mice. Physiol Behav. 2016;157:281-7. [PubMed: 26861176]

83. Luna RA, Oezguen N, Balderas M, Venkatachalam A, Runge JK, Versalovic J, et al. Distinct microbiome-Neuroimmune signatures correlate with functional abdominal pain in children with autism Spectrum disorder. Cell Mol Gastroenterol Hepatol. 2017;3(2): 218-30. [PubMed: 28275689]

84. Spees AM, Wangdi T, Lopez CA, Kingsbury DD, Xavier MN, Winter SE, et al. Streptomycininduced inflammation enhances Escherichia coli gut colonization through nitrate respiration. MBio. 2013;4(4).

85. Winter SE, Winter MG, Xavier MN, Thiennimitr P, Poon V, Keestra AM, et al. Host-derived nitrate boosts growth of E. coli in the inflamed gut. Science. 2013;339(6120):708-11. [PubMed: 23393266]

86. Rose D, Ashwood P. Potential cytokine biomarkers in autism spectrum disorders. Biomark Med. 2014;8(9):1171-81. [PubMed: 25402586]

87. Cao X, Lin P, Jiang P, Li C. Characteristics of the gastrointestinal microbiome in children with autism spectrum disorder: a systematic review. Shanghai Arch Psychiatry. 2013;25(6):342-53. [PubMed: 24991177]

88. Hiergeist A, Glasner J, Reischl U, Gessner A. Analyses of intestinal microbiota: culture versus sequencing. ILAR J. 2015;56(2): 228-40. [PubMed: 26323632]

89. Cermak SA, Curtin C, Bandini LG. Food selectivity and sensory sensitivity in children with autism spectrum disorders. J Am Diet Assoc. 2010;110(2):238-46. [PubMed: 20102851]

90. Partty A, Kalliomaki M, Wacklin P, Salminen S, Isolauri E. A possible link between early probiotic intervention and the risk of neuropsychiatric disorders later in childhood: a randomized trial. Pediatr Res. 2015;77(6):823-8. [PubMed: 25760553]

91. Cui L, Morris A, Ghedin E. The human mycobiome in health and disease. Genome Med. 2013;5(7):63. [PubMed: 23899327]

92. Li Q, Wang C, Tang C, He Q, Li N, Li J. Dysbiosis of gut fungal microbiota is associated with mucosal inflammation in Crohn's disease. J Clin Gastroenterol. 2014;48(6):513-23. [PubMed: 24275714]

93. Gerard R, Sendid B, Colombel JF, Poulain D, Jouault T. An immunological link between Candida albicans colonization and Crohn's disease. Crit Rev Microbiol. 2015;41(2):135-9. [PubMed: 23855357]

94. Harnett J, Myers SP, Rolfe M. Significantly higher faecal counts of the yeasts candida and saccharomyces identified in people with coeliac disease. Gut Pathogens. 2017;9:26. [PubMed: 28484520]

95. Erb Downward JR, Falkowski NR, Mason KL, Muraglia R, Huffnagle GB. Modulation of postantibiotic bacterial community reassembly and host response by Candida albicans. Sci Rep. 2013;3:2191. [PubMed: 23846617]

96. Kantarcioglu AS, Kiraz N, Aydin A. Microbiota-gut-brain Axis: yeast species isolated from stool samples of children with suspected or diagnosed autism Spectrum disorders and in vitro susceptibility against nystatin and fluconazole. Mycopathologia. 2016;181(1-2):1-7. [PubMed: 26442855]

97. Iovene MR, Bombace F, Maresca R, Sapone A, Iardino P, Picardi A, et al. Intestinal Dysbiosis and yeast isolation in stool of subjects with autism Spectrum disorders. Mycopathologia. 2017;182(34): 349-63. [PubMed: 27655151] 
98. Sigmundsdottir G, Larsson L, Wiebe T, Bjorklund LJ, Christensson B. Clinical experience of urine D-arabinitol/L-arabinitol ratio in the early diagnosis of invasive candidiasis in paediatric high risk populations. Scand J Infect Dis. 2007;39(2):146-51. [PubMed: 17366032]

99. Vernocchi P, Del Chierico F, Putignani L. Gut microbiota profiling: metabolomics based approach to unravel compounds affecting human health. Front Microbiol. 1144;7:2016.

100. Shultz SR, MacFabe DF, Ossenkopp KP, Scratch S, Whelan J, Taylor R, et al. Intracerebroventricular injection of propionic acid, an enteric bacterial metabolic end-product, impairs social behavior in the rat: implications for an animal model of autism. Neuropharmacology. 2008;54(6):901-11. [PubMed: 18395759]

101. MacFabe DF, Cain NE, Boon F, Ossenkopp KP, Cain DP. Effects of the enteric bacterial metabolic product propionic acid on object-directed behavior, social behavior, cognition, and neuroinflammation in adolescent rats: relevance to autism spectrum disorder. Behav Brain Res. 2011;217(1):47-54. [PubMed: 20937326]

102••. Arpaia N, Campbell C, Fan X, Dikiy S, van der Veeken J, de Roos P, et al. Metabolites produced by commensal bacteria promote peripheral regulatory T-cell generation. Nature. 2013;504(7480): 451-5 [PubMed: 24226773] Important study showing that short-chain fatty acids produced by commensal microbiota act as histone deacetylase inhibitors, promoting expression of Foxp3 and expansion of peripheral regulatory T cells.

103. Agus A, Planchais J, Sokol H. Gut microbiota regulation of tryptophan metabolism in health and disease. Cell Host Microbe. 2018;23(6):716-24. [PubMed: 29902437]

104. Wikoff WR, Anfora AT, Liu J, Schultz PG, Lesley SA, Peters EC, et al. Metabolomics analysis reveals large effects of gut microflora on mammalian blood metabolites. Proc Natl Acad Sci USA. 2009;106(10):3698-703. [PubMed: 19234110]

105. Diémé B, Mavel S, Blasco H, Tripi G, Bonnet-Brilhault F, Malvy J, et al. Metabolomics study of urine in autism Spectrum disorders using a multiplatform analytical methodology. J Proteome Res. 2015;14(12):5273-82. [PubMed: 26538324]

106. Guillemin GJ, Smith DG, Smythe GA, Armati PJ, Brew BJ. Expression of the kynurenine pathway enzymes in human microglia and macrophages. Adv Exp Med Biol. 2003;527:105-12. [PubMed: 15206722]

107. Li Z, Chalazonitis A. Huang Y-y, Mann JJ, Margolis KG, Yang QM, et al. essential roles of enteric neuronal serotonin in gastrointestinal motility and the development/survival of enteric dopaminergic neurons. J Neurosci. 2011;31(24):8998-9009. [PubMed: 21677183]

108. Ruddick JP, Evans AK, Nutt DJ, Lightman SL, Rook GA, Lowry CA. Tryptophan metabolism in the central nervous system: medical implications. Expert Rev Mol Med. 2006;8(20):1-27.

109. Miceli S, Negwer M, van Eijs F, Kalkhoven C, van Lierop I, Homberg J, et al. High serotonin levels during brain development alter the structural input-output connectivity of neural networks in the rat somatosensory layer IV. Front Cell Neurosci. 2013;7:88. [PubMed: 23761736]

110. Bonaz B, Bazin T, Pellissier S. The Vagus nerve at the Interface of the microbiota-gut-brain Axis. Front Neurosci. 2018;12:49. [PubMed: 29467611]

111. Bercik P, Park AJ, Sinclair D, Khoshdel A, Lu J, Huang X, et al. The anxiolytic effect of Bifidobacterium longum NCC3001 involves vagal pathways for gut-brain communication. Neurogastroenterol Motil. 2011;23(12):1132-9. [PubMed: 21988661]

112. Bercik P, Denou E, Collins J, Jackson W, Lu J, Jury J, et al. The intestinal microbiota affect central levels of brain-derived neurotropic factor and behavior in mice. Gastroenterology. 2011;141(2): 599-609 e1-3. [PubMed: 21683077] 


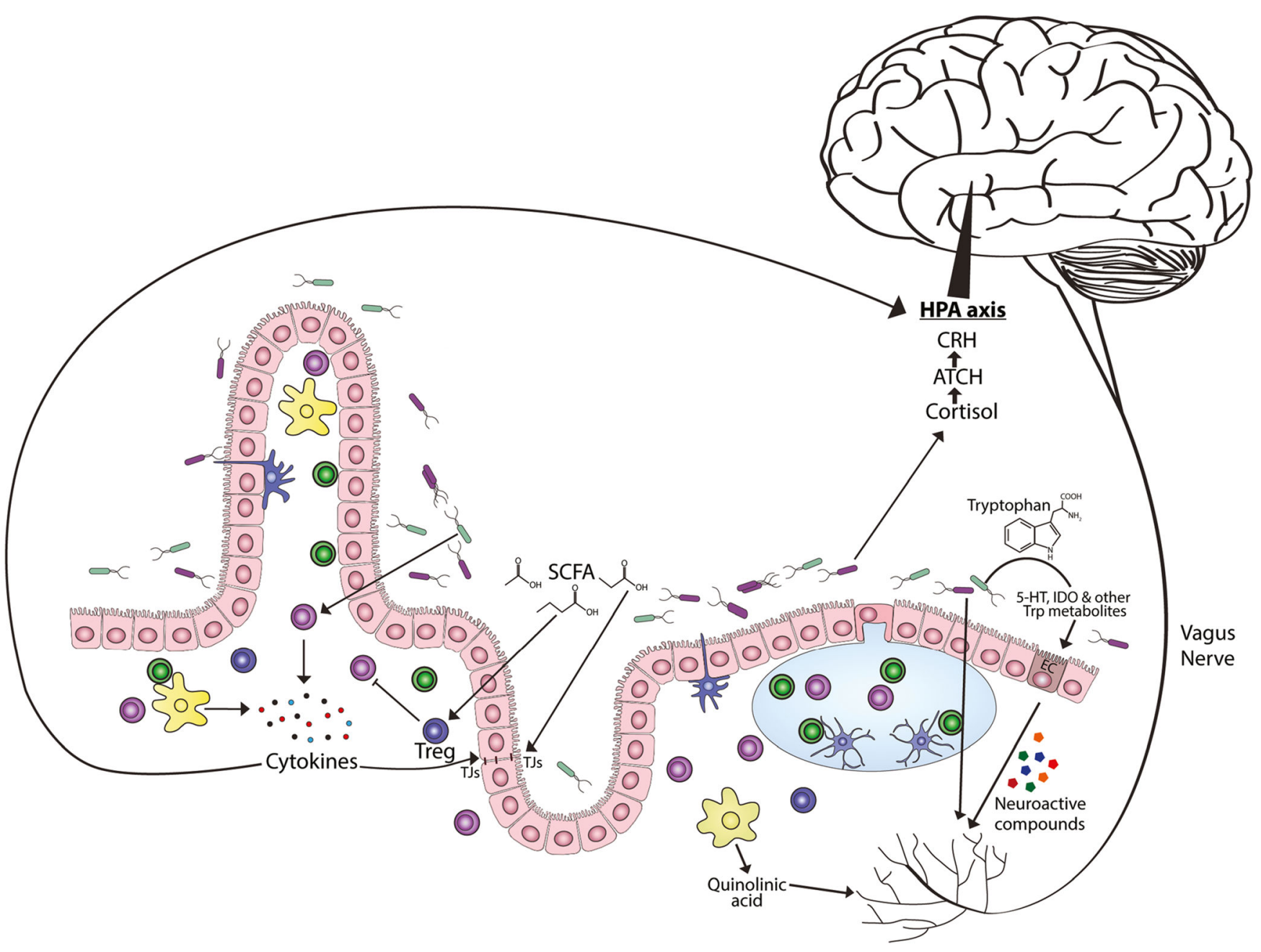

Fig. 1.

Mechanisms of the microbiota-gut-brain axis. Possible mechanisms of the MGB-axis are being actively investigated and include neuroimmune pathways, neural communication through the vagus nerve, influence of metabolites produced by the microbiota, microbialderived neurotransmitters, and the significant influence the microbiota have on tryptophan, kynurenine, and serotonin metabolism. Short-chain fatty acids (SCFA) can promote peripheral $\mathrm{T}$ regulatory (Treg) cell expansion as well as influence tight junction (TJ) proteins and intestinal barrier function. Microbiota regulate tryptophan (Trp) metabolites by degrading Trp to indole-derivatives or through kynurenine and serotonin pathways, such as increasing expression of tryptophan hydroxylase (Tph) 1 in enterochromaffin (EC) cells. Dysbiosis can promote activation of immune cells, including macrophages that produce quinolinic acid (QA) through an alternative kynurenine pathway, a known excitotoxic Nmethyl-D-aspartate (NMDA) receptor agonist. Activated immune cells also produce proinflammatory cytokines which can further disrupt microflora and impact intestinal barrier function. Neural communication can also occur through the vagus nerve via signaling from hormones and neurotransmitters release by gut endocrine cells and immune cells. Breech of 
the intestinal barrier would also allow direct pattern recognition sensing due to Toll-like receptor expression on afferent fibers 


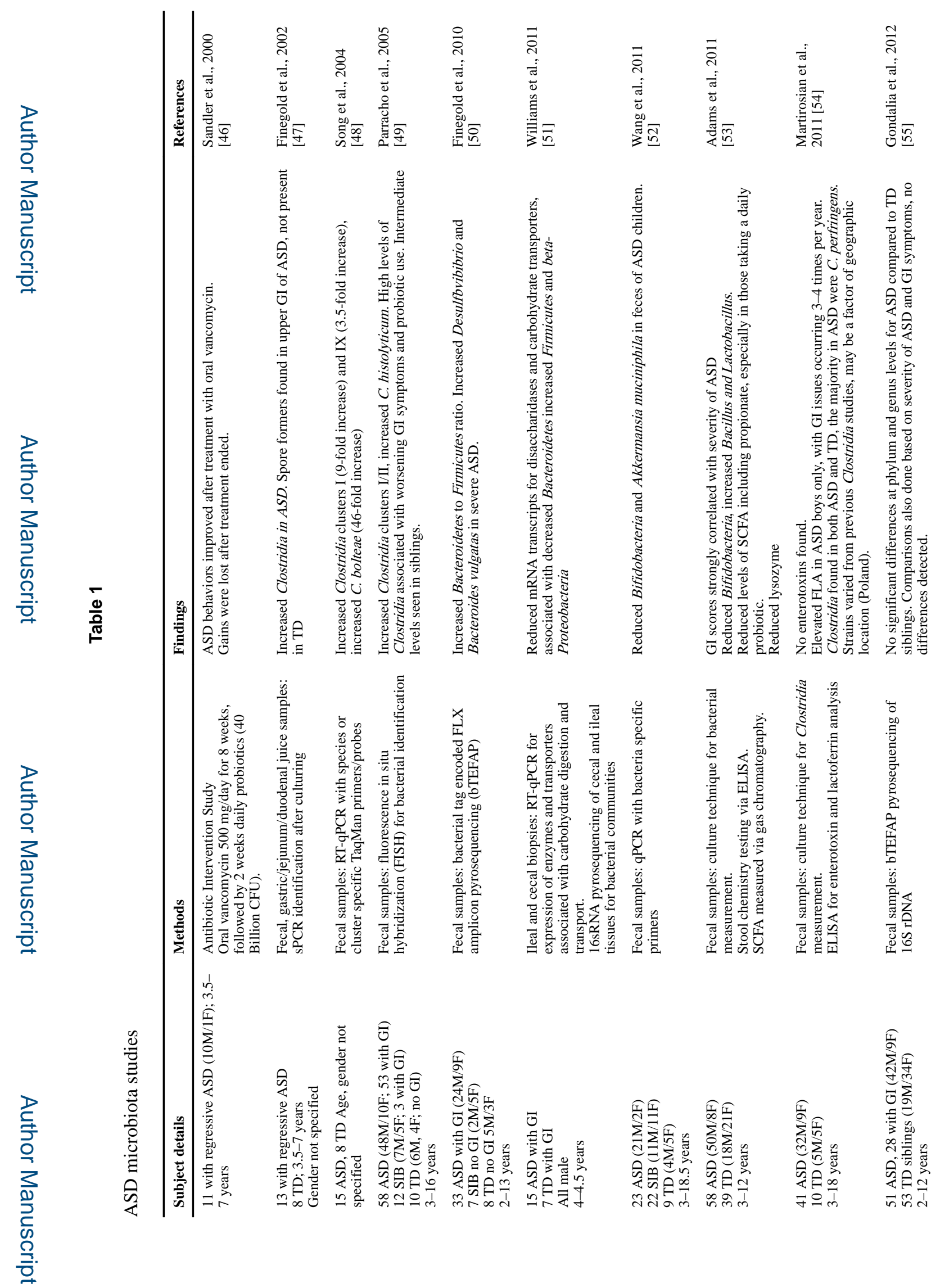

Curr Neurol Neurosci Rep. Author manuscript; available in PMC 2019 November 14. 
Hughes et al.

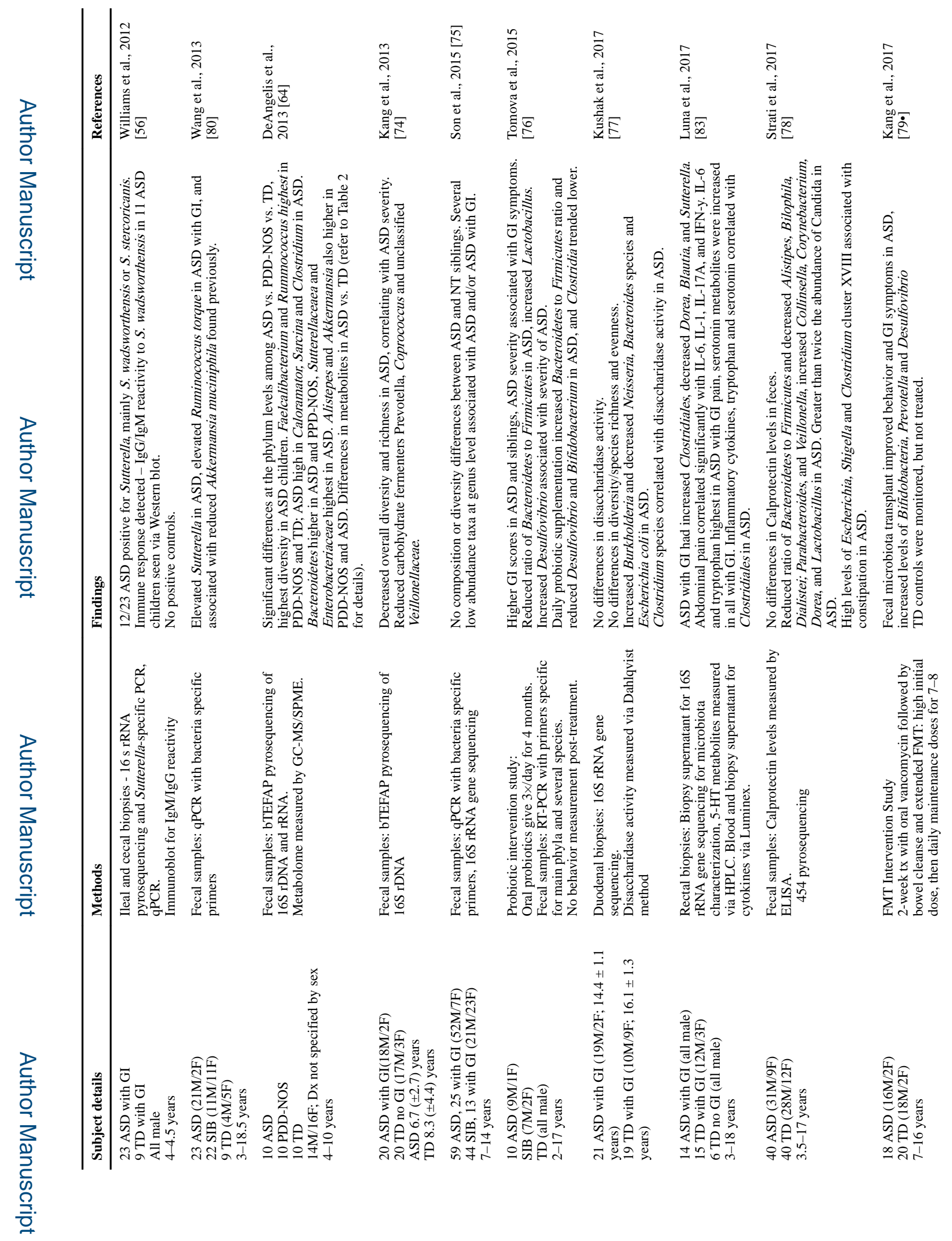

Curr Neurol Neurosci Rep. Author manuscript; available in PMC 2019 November 14. 
Hughes et al.

Page 20

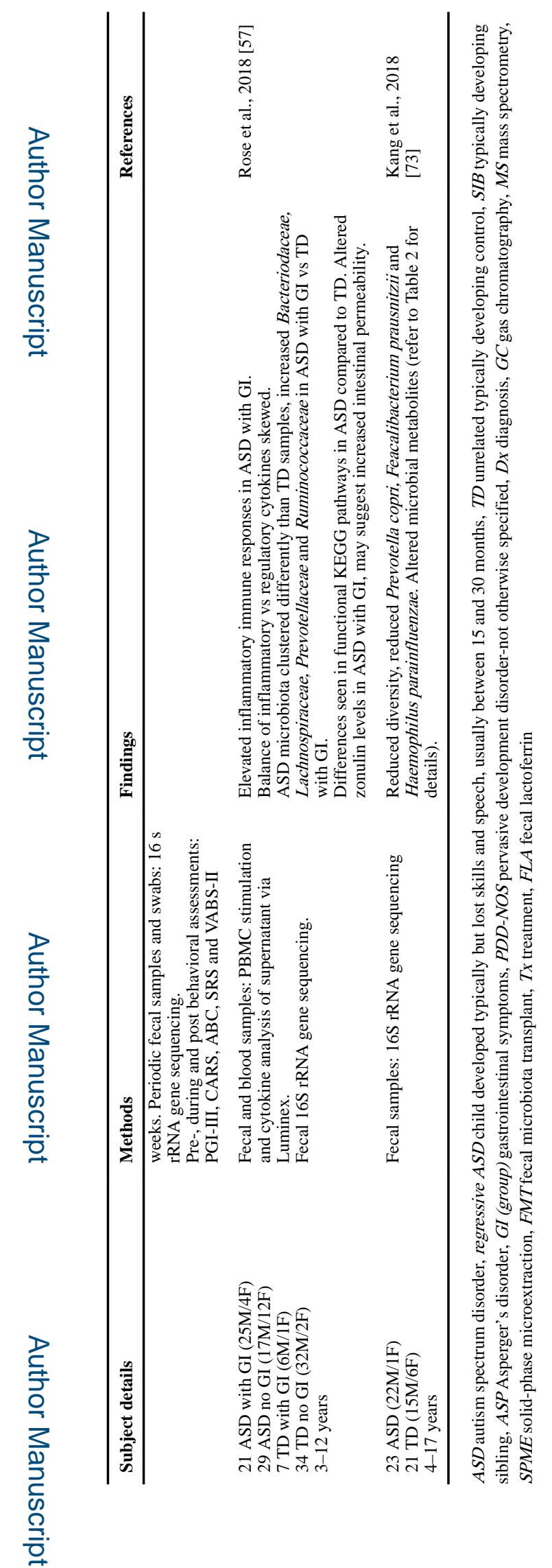

Curr Neurol Neurosci Rep. Author manuscript; available in PMC 2019 November 14. 


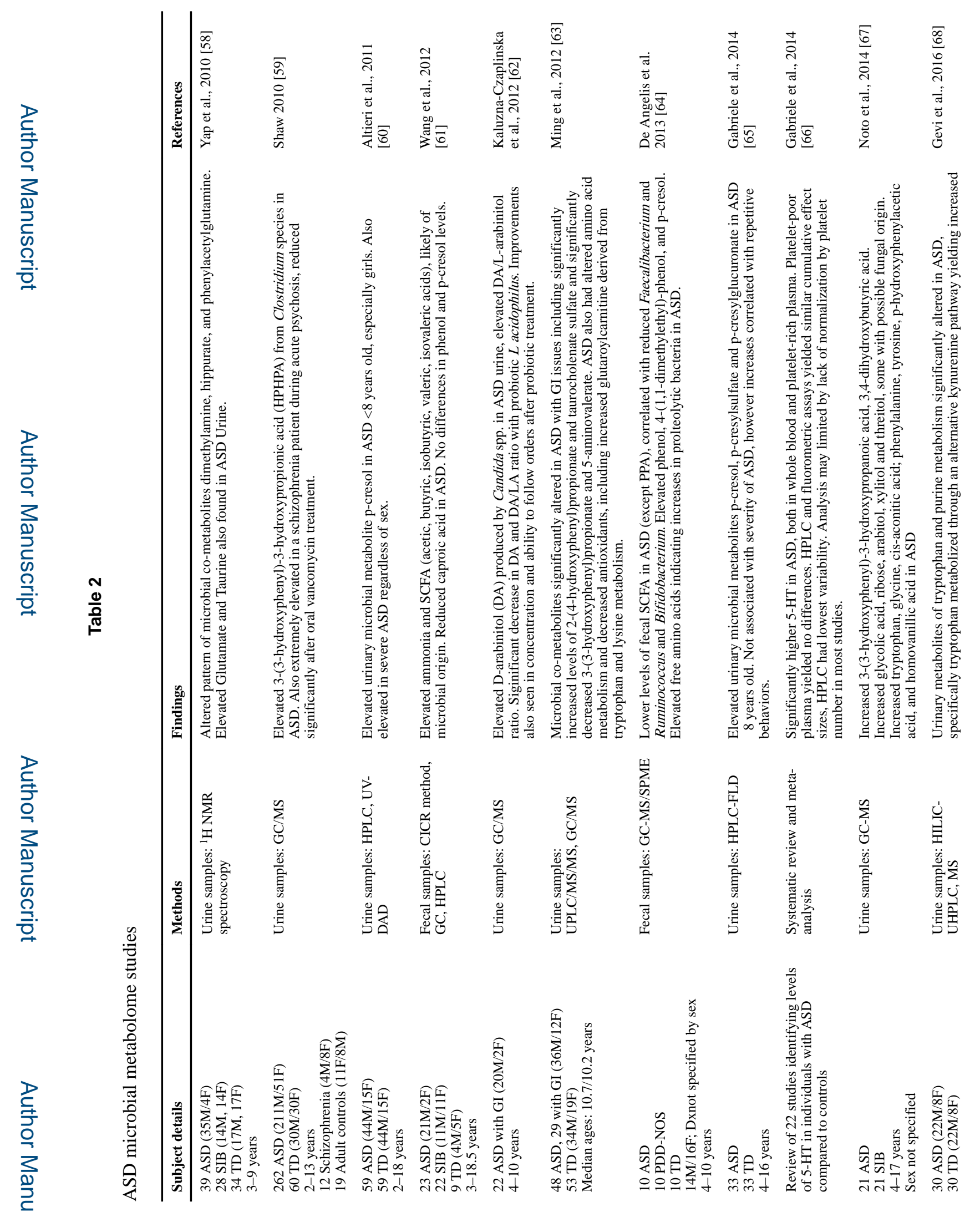

Curr Neurol Neurosci Rep. Author manuscript; available in PMC 2019 November 14. 
Hughes et al.

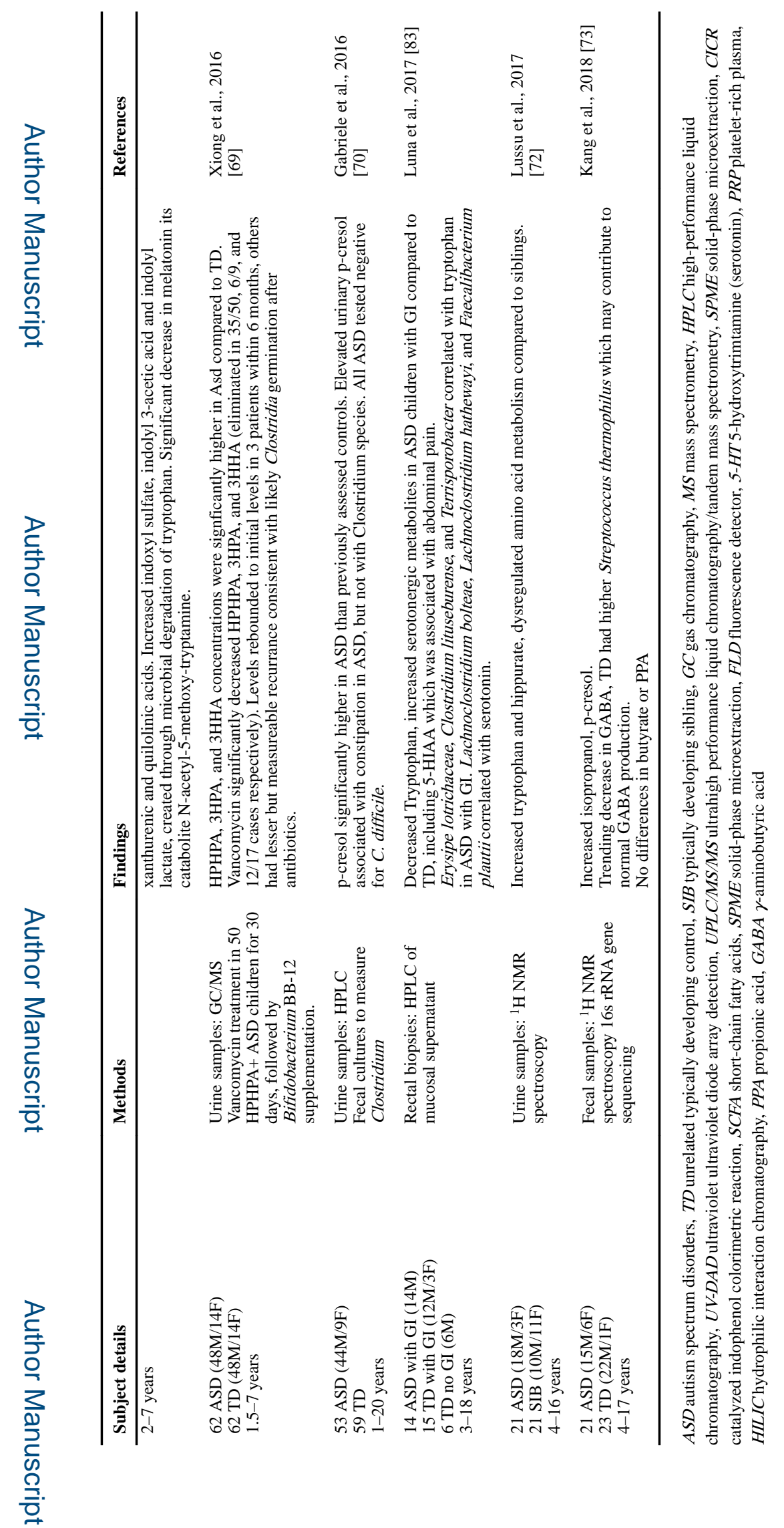

Curr Neurol Neurosci Rep. Author manuscript; available in PMC 2019 November 14. 